\title{
Timp-3 deficiency impairs cognitive function in mice
}

\author{
Yoshichika Baba ${ }^{1}$, Osamu Yasuda', Yukihiro Takemura', Yasuyuki Ishikawa ${ }^{2}$, Mitsuru Ohishi ${ }^{1}$, Jun Iwanami ${ }^{3}$, \\ Masaki Mogi ${ }^{3}$, Nobutaka Doe ${ }^{4}$, Masatsugu Horiuchi ${ }^{3}$, Nobuyo Maeda ${ }^{5}$, Keisuke Fukuo ${ }^{6}$ and Hiromi Rakugi ${ }^{1}$
}

Extracellular matrix (ECM) degradation is performed primarily by matrix metalloproteinases (MMPs). MMPs have recently been shown to regulate synaptic activity in the hippocampus and to affect memory and learning. The tissue inhibitor of metalloproteinase (Timp) is an endogenous factor that controls MMP activity by binding to the catalytic site of MMPs. At present, four Timp isotypes have been reported (Timp-1 through Timp-4) with 35-50\% amino-acid sequence homology. Timp-3 is a unique member of Timp proteins in that it is bound to the ECM. In this study, we used the passive avoidance test, active avoidance test, and water maze test to examine the cognitive function in Timp-3 knockout (KO) mice. Habituation was evaluated using the open-field test. The water maze test showed that Timp-3 KO mice exhibit deterioration in cognitive function compared with wild-type (WT) mice. The open-field test showed decreased habituation of Timp-3 KO mice. Immunostaining of brain slices revealed the expression of Timp-3 in the hippocampus. In situ zymography of the hippocampus showed increased gelatinolytic activity in Timp-3 KO mice compared with WT mice. These results present the first evidence of Timp-3 involvement in cognitive function and hippocampal MMP activity in mice. Moreover, our findings suggest a novel therapeutic target to be explored for improvement of cognitive function in humans.

Laboratory Investigation (2009) 89, 1340-1347; doi:10.1038/labinvest.2009.101; published online 5 October 2009

KEYWORDS: cognitive function; extracellular matrix; hippocampus; matrix metalloproteinase; Timp-3

Extracellular matrix (ECM) molecules have important roles in the structural changes of brain synapses involved in neural plasticity, learning, and memory. ${ }^{1}$ ECM interacts with cells through cell surface receptors, such as integrin, cadherin, and neural adhesion molecules, ${ }^{2}$ and these interactions affect cell proliferation, growth, migration, synaptic stabilization, and apoptosis. Thus, the ECM develops a wide range of signals within the brain tissue. ${ }^{3}$

Matrix metalloproteinases (MMPs) comprise a family of protein-digesting enzymes that have an important role in structural maintenance and conversion of the ECM. ${ }^{4}$ MMPs target many substrates, including proteases, growth factors, cytokines, cell surface receptors, and cell adhesion molecules. $^{5}$ Excess activation of MMPs occurs under several pathophysiological conditions, such as rheumatoid arthritis and rupture of atherosclerotic plaques. ${ }^{6-8}$ Thus, MMP activity is tightly controlled at the level of transcription, activation of the precursor zymogens, and inhibition by the tissue inhibitors of metalloproteinase (Timp). ${ }^{9}$

At present, four members of the Timp family (Timp-1 to Timp-4), possessing 35-50\% amino-acid sequence homology, have been identified. All Timp isotypes contain $12 \mathrm{cy}$ steines that form 6 disulfide bonds. To inhibit MMP activity, Timp proteins form a 1:1 complex with a zinc-binding site in the catalytic region of MMP. ${ }^{10}$

Each Timp protein has unique characteristics. Timp-1, Timp-2, and Timp- 4 are present in soluble form. ${ }^{11-13}$ Timp3 , which is tightly bound to the ECM, is involved in cell proliferation, apoptosis, and angiogenesis. ${ }^{1-13}$ Timp-1 binds to proMMP-9, Timp-2 binds to proMMP-2, and Timp-3 binds to both proMMP-2 and proMMP-9. ${ }^{13,14}$

In the central nervous system, the MMP/Timp system is responsive to changes in neural activity. ${ }^{15}$ Deregulation of MMP activity is involved in various neurological diseases,

\footnotetext{
${ }^{1}$ Department of Geriatric Medicine, Osaka University Graduate School of Medicine, Suita, Osaka, Japan; ${ }^{2}$ Division of Structural Cell Biology, Nara Institute of Science and Technology, Takayama-cho, Ikoma, Nara, Japan; ${ }^{3}$ Department of Molecular Cardiovascular Biology and Pharmacology, Ehime University, Graduate School of Medicine, Shitsukawa, Tohon, Ehime, Japan; ${ }^{4}$ Section of Behavioral Science, Kouiken Co. Ltd., Akashi, Hyogo, Japan; ${ }^{5}$ Department of Pathology and Laboratory Medicine, School

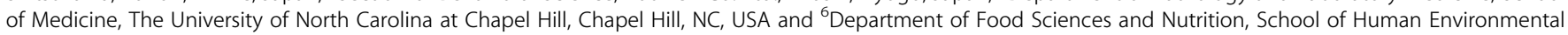
Sciences, Mukogawa Woman's University, Nishinomiya, Hyogo, Japan

Correspondence: Dr O Yasuda, MD, PhD, Department of Geriatric Medicine, Osaka University Graduate School of Medicine, 2-2 Yamadaoka, Suita, Osaka 565-0871, Japan.
}

E-mail: yasuda@geriat.med.osaka-u.ac.jp

Received 16 February 2009; revised 27 July 2009; accepted 20 August 2009 
including multiple sclerosis, infection with human immunodeficiency virus, and spinal cord injury. ${ }^{16-18}$ Moreover, MMPs are implicated in the invasion of malignant glioma cells into the brain parenchyma. ${ }^{19}$

In Alzheimer's disease, MMP inhibits angiogenesis and accumulation of amyloid- $\beta .{ }^{16,20,21}$ Recently, MMPs have been reported to control synaptic activity in the hippocampus and to affect learning and memory. ${ }^{22}$ Other studies have clarified the contributions of Timp-1 and Timp-2 in learning and memory. Mice deficient for Timp-1 or Timp-2 exhibit defective memory function. ${ }^{23-25}$ Conversely, mice overexpressing Timp-1 showed a slight, but significant, improvement in learning and memory. ${ }^{24}$ However, there is no published evidence for a role of Timp-3 in the regulation of cognitive function.

This study investigated the effects of Timp-3 on learning and memory. We conducted various behavioral tests with wild-type (WT) and Timp-3 knockout (KO) mice, and further examined the expression of Timp-3 and compared gelatinolytic activity in WT and KO brain tissues.

\section{MATERIALS AND METHODS Timp-3 KO Mice}

Timp-3 KO mice were produced using the gene-targeting technique described by Kawamoto et al. ${ }^{26}$ Briefly, mice carrying the mutant allele were backcrossed with $\mathrm{C} 57 \mathrm{BL} / 6$ mice to generate $\mathrm{KO}$ mice in a C57BL/6 background. Genotyping of mice was performed by PCR using tail DNA. ${ }^{26}$

\section{Experimental Conditions for Behavioral Tests}

All behavioral tests were conducted in the laboratory at $22{ }^{\circ} \mathrm{C}$ and 55\% (50-60\%) humidity. Illumination for the experimental device was set at $250 \mathrm{~lx}$. The ventilation fan provided a masking noise of $40 \mathrm{~dB}$, which was deemed appropriate for behavioral tests.

Mice were maintained in individual acrylic cages and naturalized to the environment during the 3 days before testing. They were given access to dry, solid feed (Labo MR Stock from Nihon Nosan, Yokohama, Japan) ad libitum. The room was maintained on a 12-h light and dark cycle, with the light cycle starting at 0800 hours and ending at 2000 hours. All tests were started after 1000 hours and conducted during the light period.

The passive avoidance test and water maze test with an invisible platform were conducted with 12 male WT mice and $12 \mathrm{KO}$ mice. The open-field habituation test, active avoidance test, and water maze test with a visible platform were conducted using a different set of 12 male WT mice and $12 \mathrm{KO}$ mice.

The experimental protocols were approved by the Osaka University Medical School Animal Care and Use Committee, and performed according to the Osaka University Medical School Guidelines for the Care and Use of Laboratory Animals.

\section{Passive Avoidance Test}

An avoidance-learning box was constructed, with a lighted chamber $\left(15 \times 15 \times 20 \mathrm{~cm}^{3}\right)$ and dark chamber $\left(15 \times 15 \times 20 \mathrm{~cm}^{3}\right)$ connected to each other. A guillotine door separated the two compartments. A mouse was placed in the lighted chamber, and the guillotine door was opened. When the mouse spontaneously moved into the dark chamber, the guillotine door was closed. Within $10 \mathrm{~s}$, a 3-s, $160-\mathrm{V}$ AC electrical shock was delivered through the grid floor. The latency period for the mouse to enter the dark chamber was recorded. After this single learning trial, the mouse was immediately removed from the device.

After $24 \mathrm{~h}$, the same mouse was put in the lighted chamber for the single retention test. The latency period ( $\leq 300 \mathrm{~s}$ ) for the mouse to enter the dark chamber was recorded. No electric shock was given during the retention test.

\section{Active Avoidance Test}

An avoidance-learning box with two connecting compartments (each $15 \times 15 \times 20 \mathrm{~cm}^{3}$ ) was constructed. The mouse was able to move freely between the two compartments. To detect movement, two infrared ray beams were attached on both walls on the sides of each compartment, $2 \mathrm{~cm}$ above the floor and $5 \mathrm{~cm}$ from the gate. The avoidance-learning box, which was placed in a ventilated, sound-attenuating chamber to maintain a background noise level of $64 \mathrm{~dB}$ throughout the session, was indirectly illuminated by white bulbs fixed to the ceiling of the chamber. A $1500-\mathrm{Hz}$ pure tone with $85 \mathrm{~dB}$ of sound pressure was used as a conditioned stimulus (CS), and a 140-V AC electrical shock delivered from the grid floor was used as an unconditioned stimulus (US).

A mouse was placed in one compartment and allowed to move between the two compartments throughout the training session. The US, which was delivered from the grid floor $5 \mathrm{~s}$ after the CS was delivered, overlapped for a maximum of $15 \mathrm{~s}$. When the mouse moved to the connecting compartment within $5 \mathrm{~s}$ from the time of the CS, the US was not delivered, and the movement of the mouse was counted as avoidance behavior. When the mouse failed to move to the connecting compartment, the US was delivered. Both the CS and US were terminated immediately when the mouse moved to the connecting compartment after the onset of the US. The number of migration reactions in inter-trial intervals $(25 \mathrm{~s}$ on average) was measured as an indicator of spontaneous activity. This active avoidance test was performed for 3 consecutive days, with one 50-trial session per day.

\section{Water Maze Test}

The water maze test was conducted using a round pool (inside diameter $=95 \mathrm{~cm}$; depth $=35 \mathrm{~cm}$ ) filled with water, made opaque by the addition of titanium oxide to a depth of $22 \mathrm{~cm}$. The temperature of the water was maintained at $22 \pm 1{ }^{\circ} \mathrm{C}$ using a thermostatic heater. The pool was set on a pedestal $(30 \mathrm{~cm}$ in height), and was enclosed $\left(\right.$ area $\left.=130 \times 130 \mathrm{~cm}^{2}\right)$ by four white walls $(120 \mathrm{~cm}$ in 
height). As extra-maze cues, letter-sized posters, a CCD camera, and a black doll $(20 \mathrm{~cm}$ in height $)$ were attached to the walls.

In the invisible platform test, a clear, round platform (diameter $=10 \mathrm{~cm}$ ) was submerged $0.5 \mathrm{~cm}$ below the surface of the water. Training trials were conducted for 5 consecutive days, with 5 trial sessions per day. In the three quadrants away from the platform, a mouse placed near the wall was released into the pool. The releasing quadrants varied with pseudo-random sequences for each mouse. The escape latency period (measured for a maximum of $60 \mathrm{~s}$ ) was defined as the time it took for a mouse to reach the platform. The training trial terminated when the mouse reached the platform and remained on it for $10 \mathrm{~s}$. In cases in which the mouse did not find the platform within $60 \mathrm{~s}$, the mouse was guided to the platform by the experimenter and was kept on the platform for $10 \mathrm{~s}$. On the day after 5 consecutive days of access training, the platform was removed, and a 1-min probe test was performed. The 1-min probe test measured latency time in the quadrant in which the platform was previously placed.

The visible platform test was conducted independently using a different group of $12 \mathrm{WT}$ and $12 \mathrm{KO}$ mice (ie, different groups of mice from those used in the invisible platform test). Methodology for the visible platform test was similar, except (1) the platform was not submerged, but rather was placed $0.5 \mathrm{~cm}$ above the surface of the water, and (2) as a cue, a stick with a black cube on top was placed on the platform.

\section{Open-Field Habituation Test}

The apparatus for the open-field habituation test was manufactured by Taiyo Electric. (Tokyo, Japan). WT and KO mice were placed in an acrylic box $\left(30 \times 30 \times 30 \mathrm{~cm}^{3}\right)$ stored within a ventilated, soundproof chamber. An incandescent bulb, which was fixed to the ceiling of the chamber, provided lighting of $\sim 110 \mathrm{~lx}$ in the chamber. A fan attached to the wall of the chamber produced a masking noise of $45 \mathrm{~dB}$. Habituation of the mice to the environment was measured as a function of locomotion and rearing behavior. The number of episodes of locomotion and rearing behavior, which were recorded with infrared ray beams placed on the lateral side of the box, was scored. The open-field habituation test was conducted for 3 days, with one 10-min session per day.

\section{Reverse Transcription-PCR}

RNA was isolated from mouse hippocampus using the ISOGEN (Nippon Gene, Tokyo, Japan) kit, according to the manufacturer's instructions. Timp-3 gene expression was detected by reverse transcribing the isolated RNA and amplifying the product with PCR (RT-PCR). The PCR amplification was performed with the following primers: Timp-3: Timp-3F, $5^{\prime}$-CACGGAAGCCTCTGAAAGTC- ${ }^{\prime}$, and Timp-3R, 5'-CCCAAAATTGGAGAGCATGT-3'. GAPDH: GAPDH-F,
5'-AAATGGTGAAGGTCGGTGTG-3' ${ }^{\prime}$, and GAPDH-R, $5^{\prime}$-GC AGAAGGGGCGGAGATGAT- ${ }^{\prime}$.

\section{Immunostaining}

WT and Timp-3 KO mice were killed, and their brains were collected, fixed with formaldehyde, embedded in paraffin, and cut into $10-\mu \mathrm{m}$-thick sections with a microtome. To remove endogenous peroxidase, the sections were treated with $0.3 \%$ hydrogen peroxide in methanol at room temperature. The sections were washed with $0.05 \mathrm{M}$ phosphate buffer ( $\mathrm{pH}$ 7.6) thrice for $3 \mathrm{~min}$. The sections were then treated with phosphate buffer containing $0.5 \%$ bovine serum albumin and $0.1 \%$ sodium azide for $10 \mathrm{~min}$ at room temperature. This was performed to absorb nonspecific proteins. Subsequently, the sections were allowed to react with the anti-Timp-3 rabbit polyclonal antibody (ProteinTech, Chicago, IL, USA) $(500 \times$ dilution $)$ overnight at $4{ }^{\circ} \mathrm{C}$. Next, the sections were washed thrice for $3 \mathrm{~min}$ with $0.05 \mathrm{M}$ phosphate buffer ( $\mathrm{pH}$ 7.6), and then allowed to react for $30 \mathrm{~min}$ with the secondary antibody conjugated with peroxidase. Thereafter, the sections were again washed with $0.05 \mathrm{M}$ phosphate buffer. After a 5-min chromogenic reaction with 3,3'-Diaminobenzidine $\mathrm{HCl}$, the sections were counterstained with Mayer's hematoxylin for $5 \mathrm{~min}$. The sections were then dehydrated and encapsulated for observation under an optical microscope.

\section{In Situ Zymography}

Gelatinolytic activity in mouse brain sections was determined by in situ zymography with DQ-gelatin-FITC (Molecular Probes, Eugene, OR, USA) as described previously. ${ }^{27,28}$ Briefly, unfixed whole mouse brain was embedded in OCT compound. Sections (of $10-\mu \mathrm{m}$ thickness) were cut and air dried for $1 \mathrm{~h}$, re-hydrated in PBS, and incubated at $37^{\circ} \mathrm{C}$ in DQ-gelatin-FITC solution (100 $\mu \mathrm{g} / \mathrm{ml}$ in PBS) for $1 \mathrm{~h}$. Sections were then washed thrice in PBS, fixed in $4 \%$ paraformaldehyde, and examined under a fluorescent microscope to detect green fluorescence due to gelatinolytic activity.

\section{Statistical Analysis}

Results are expressed as mean \pm s.e. Comparison among groups was performed by one-way ANOVA; Student's $t$-test was used when appropriate. A value of $P<0.05$ was considered significant.

\section{RESULTS}

\section{Passive Avoidance Test}

WT and KO mice first underwent preconditioning. When a mouse moved from the lighted chamber into the dark chamber, it received an electric shock. Twenty-four hours after preconditioning, one retention trial was performed. In the retention trial, the latency time in the lighted chamber was measured and compared between the two mice groups. As a result of receiving an electric shock in the dark chamber, the mean latency time in the retention trial was prolonged in 
both WT and KO mice, indicating that both types of mice acquired avoidance memory. Moreover, no significant difference was observed in latency times for WT and KO mice, either before $(54.4 \pm 19.5 \mathrm{~s}$ for WT, $27.7 \pm 7.5 \mathrm{~s}$ for KO; $P=0.20)$ or after $(300 \pm 0.0 \mathrm{~s}$ for WT, $276 \pm 18.7 \mathrm{~s}$ for KO; $P=0.23)$ conditioning. These results show that there were no memory deficits in KO mice compared with WT mice.

\section{Active Avoidance Test}

WT and KO mice were placed in the avoidance-learning box with two compartments connected. A beep was presented, and $5 \mathrm{~s}$ later, an electronic shock was given through the grid floor. When this process was repeated, the mice learned to escape the shock by migrating into the adjoining compartments at the sound of the beep. A migration reaction within $5 \mathrm{~s}$ from the beep was defined as the 'avoidance' reaction. The number of avoidance reactions was counted over 3 days, with 50 trials per day. Moreover, the number of migrations between trial intervals in the absence of stimuli was counted to compare general activity levels.

We found no differences in activity levels between WT and KO mice over the 3 days of testing $(\mathrm{F}(1,22)=1.88$, $P=0.183)$. Successful avoidance increased in both WT and $\mathrm{KO}$ mice as a function of training days, indicating that both types of mice acquired avoidance memory. Furthermore, there was no difference between the number of avoidance reactions in $\mathrm{WT}$ and $\mathrm{KO}$ mice during the 3 days of testing $(\mathrm{F}(1,22)=0.46, P=0.505)$. These results indicate that no memory deficits were detected in $\mathrm{KO}$ mice.

\section{Water Maze Test with Invisible Platform}

The water maze test with the invisible platform was conducted to evaluate spatial memory in WT and KO mice. Each mouse underwent 5 access tests daily for 5 consecutive days. Escape latency period was defined as the time (measured for a maximum of $60 \mathrm{~s}$ ) it took for each mouse to reach the platform submerged in the pool of opaque water.

On the first day, no significant difference was observed between WT and KO mice. However, on the second day, memory acquisition in $\mathrm{KO}$ mice was significantly reduced compared with WT mice. Although a significant difference in memory acquisition was also observed on the third and fourth days, the difference gradually decreased (Figure 1a), and the difference in memory acquisition was no longer significant on day 5 .

To measure the time spent in the quadrant in which the platform had been previously placed, a 1-min probe test was conducted the day after (ie, on day 6) completion of the access tests. The time spent in the target quadrant was longer than that achieved merely by chance, indicating that both types of mice acquired memory of the previous location of the platform. Moreover, no significant difference was observed in the time that WT or KO mice spent in the target quadrant $(19.6 \pm 1.71 \mathrm{~s}$ for WT; $20.9 \pm 1.27 \mathrm{~s}$ for $\mathrm{KO}$; $P=0.561)$. a
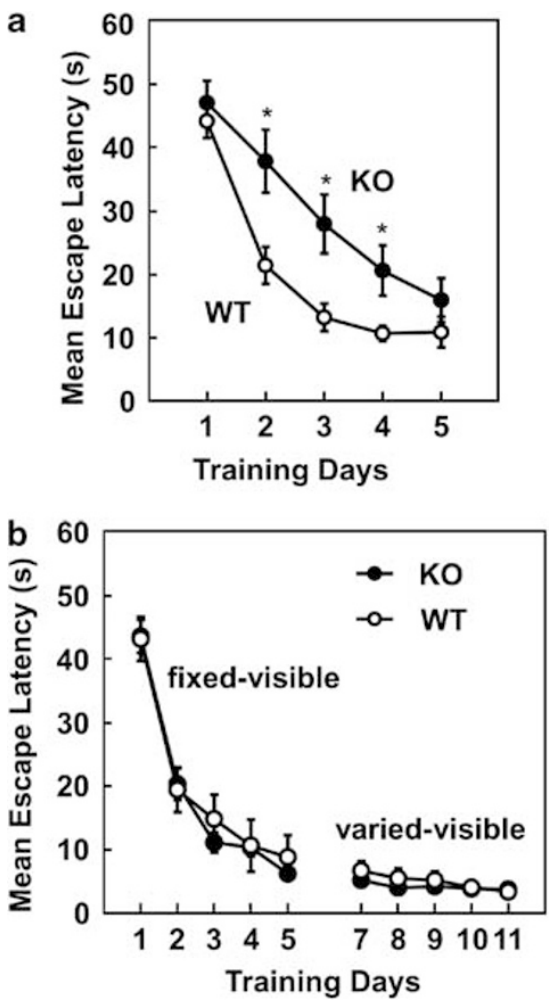

Figure 1 Water maze tests. (a) Invisible platform test of wild-type (WT; $n=12$ ) and Timp-3 knockout (KO; $n=12$ ) mice. A clear, round platform was submerged $0.5 \mathrm{~cm}$ beneath the surface of milk-colored water in a pool. Escape latency period is the time (measured for a maximum of $60 \mathrm{~s}$ ) it took for each mouse entering the pool to reach the submerged, invisible platform. Training trials were performed for 5 consecutive days, with 5 trial sessions per day. Data are expressed as mean \pm s.e. ${ }^{*} P<0.05$. (b) Visible platform test of WT $(n=12)$ and Timp-3 KO $(n=12)$ mice. The platform was placed $0.5 \mathrm{~cm}$ above the surface of water in a pool. As a visual cue, a stick with a black cube on top was placed on the platform. The location of the platform was fixed from day 1 through day 5, and varied from day 7 through day 11. Escape latency period (measured for a maximum of $60 \mathrm{~s}$ ) is the time it took for each mouse, after entering the pool, to reach the visible platform. Five trial sessions were performed per day. Data are expressed as mean \pm s.e.

Taken together, these results indicate that although KO mice eventually learned the location of the platform, their speed in acquiring memory was significantly slower than that of WT mice.

\section{Water Maze Test with Visible Platform}

In humans, a Timp-3 mutation causes Sorsby's fundus dystrophy (SFD), a disease characterized by the loss of central vision during the fourth or fifth decade of life. It is likely that the KO mice used in this study were able to recognize visual cues, given that they eventually learned the location of the platform. This conclusion is supported by a previous study showing that learning in water maze tests relies on the use of visual cues. ${ }^{29}$ To further investigate whether $\mathrm{KO}$ mice were able to use visual cues during a water maze test, a visible platform test was conducted. 
The mean escape latency period gradually decreased from day 1 to day 5 in both WT and KO mice, indicating that both types of mice acquired memory of the location of the platform (Figure 1b). Mean escape latency times for WT and KO mice were similar during the 5 days of testing. This finding indicates that both types of mice approached the platform aided by the visible cue, rather than by spatial memory. Moreover, the results suggest that the swimming ability of $\mathrm{KO}$ mice was not compromised. Moreover, latency times of WT and $\mathrm{KO}$ mice were not different, even when the location of the visible platform was varied (on days 7 through 11). The fact that latency time at day 7 , the first day in the varied visible platform test, was not longer than that at day 5 , the last day in the fixed visible platform test, also indicates that both types of mice approached the platform aided by the visual cue rather than by spatial memory.

Probe tests conducted on day $6(13.0 \pm 1.40 \mathrm{~s}$ for WT; $15.4 \pm 0.97 \mathrm{~s}$ for $\mathrm{KO} ; P=0.162)$ and day $12(12.5 \pm 1.18 \mathrm{~s}$ for WT; $12.9 \pm 0.98 \mathrm{~s}$ for KO; $P=0.821)$ also showed no difference in latency times between WT and KO mice. Furthermore, latency times observed for both types of mice were not longer than the latency times expected if achieved merely by chance. These results also indicate that movement of mice in
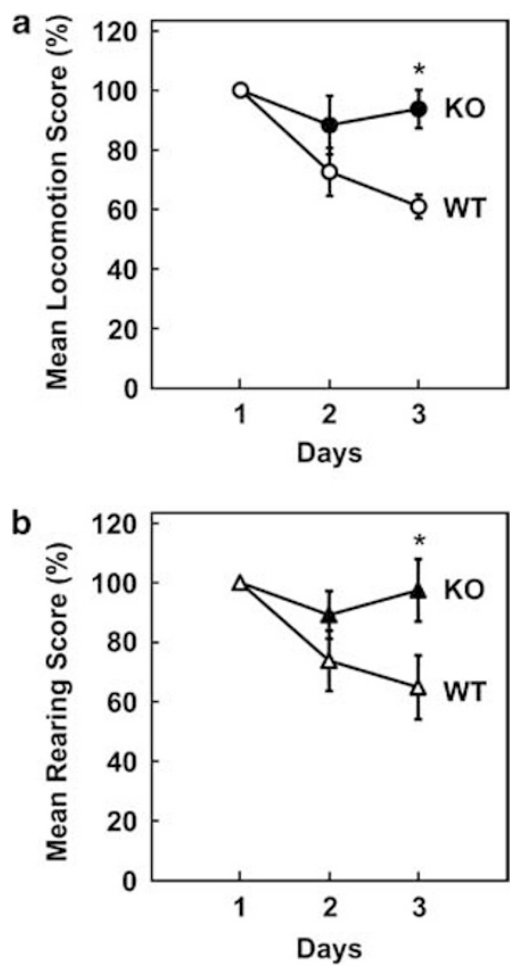

Figure 2 Open-field habituation test. (a) The graph shows the change in locomotion score for WT $(n=12)$ and Timp-3 KO $(n=12)$ mice over time (days). The locomotion score, which represents the number of migrations of each mouse in a period of $10 \mathrm{~min}$, is expressed as a percentage. Data are expressed as mean \pm s.e. ${ }^{*} P<0.05$. (b) The graph shows changes in rearing score in WT $(n=12)$ and Timp-3 KO $(n=12)$ mice over time (days). The rearing score, which represents the number of times that each mouse rose (ie, exhibited rearing behavior) in a period of $10 \mathrm{~min}$, is expressed as a percentage. Data are expressed as mean \pm s.e. ${ }^{\star} P<0.05$. the visible platform test was dependent on the use of visual cues, rather than on memory of the location of the platform.

Taken together, our findings suggest that $\mathrm{KO}$ mice were able to recognize visual cues in the water maze test. An alternative interpretation is that visual failure in $\mathrm{KO}$ mice was minimal and did not substantially compromise the recognition of visual cues.

\section{Open-Field Habituation Test}

To evaluate the motion and mobility of the mice, the numbers of locomotion and rearing were scored for $10 \mathrm{~min}$ per day for 3 days with sensors placed on the lateral side of the chamber. There was no significant difference between WT and $\mathrm{KO}$ mice in the numbers of locomotion (107.8 \pm 5.76 in WT, $116.9 \pm 10.76$ in KO; $P=0.490)$ and rearing $(99.2 \pm 6.95$ in $\mathrm{WT}, 91.6 \pm 9.19$ in $\mathrm{KO} ; P=0.535)$ on day 1 ; however, significant differences were observed on day 3 (Figure $2 \mathrm{a}$ and b). Both locomotion and rearing decreased over time in WT mice, but did not substantially change over time in KO mice. This indicated that WT, but not KO, mice became accustomed to the environment.

\section{Timp-3 Expression in the Brain}

RT-PCR showed the expression of Timp-3 in the hippocampus of WT mice (Figure 3a). In contrast, no Timp-3 was

a
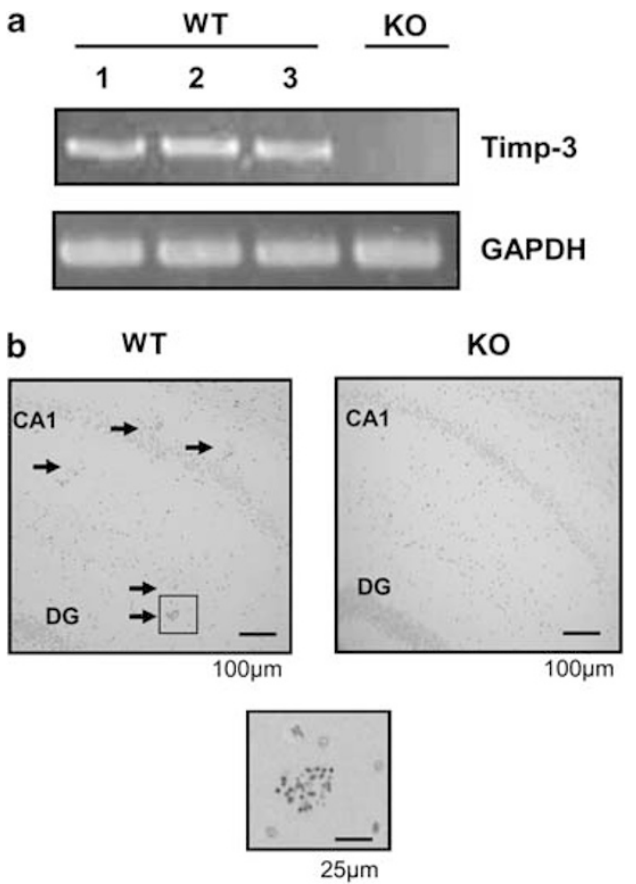

Figure 3 Expression of Timp-3 in the hippocampus. (a) RT-PCR of the Timp-3 gene. RT-PCR was performed with RNA isolated from the hippocampus of wild-type (WT) and three different Timp-3 knockout (KO) mice.

Amplification of the glyceraldehyde-3-phosphate dehydrogenase gene (GAPDH) served as a control. (b) Immunostaining of Timp-3. A Timp-3 antibody was hybridized to mouse brain sections and visualized with $3-3^{\prime}$ diaminobenzidine and a hematoxylin counterstain. Timp-3 expression is indicated in the hippocampus with arrows. The boxed region shown below is magnified $\times 4$ to illustrate Timp-3 staining. DG, dentate gyrus. 


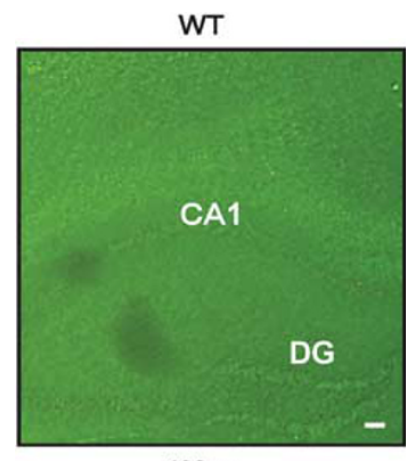

$100 \mu \mathrm{m}$

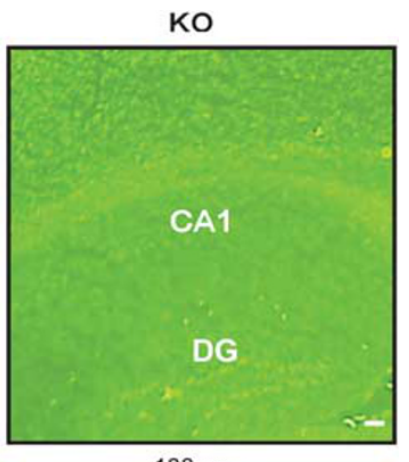

$100 \mu \mathrm{m}$
Figure 4 In Situ zymography. In situ zymography shows the gelatinolytic activity (green fluorescence) of enzymes in the brains of wild-type (WT) and Timp-3 knockout (KO) mice. Representative photographs are shown. DG, dentate gyrus.

detected in the hippocampus of KO mice. Timp-3 expression was confirmed by immunostaining brain slices. Timp-3 was expressed in the choroid plexus (not shown) and in the hippocampus (Figure $3 \mathrm{~b}$ ). This was consistent with the results from the water maze test, because the hippocampus is considered to be directly involved in memory process.

\section{In Situ Zymography}

In situ zymography showed that gelatinolytic activity was enhanced in the hippocampus of KO mice compared with that of WT mice. This result was reproducible in four independent experiments. Data from a representative experiment are shown in Figure 4. This result indicated that gelatinolytic enzyme activity was more active in the $\mathrm{KO}$ than in WT mice brains.

\section{DISCUSSION}

The ECM in a normal, healthy tissue is maintained by a balance of synthesis and degradation, and has a significant role in maintaining tissue homeostasis. In the central nervous system, the balance between Timp and MMP is believed to be involved in synaptic plasticity, particularly in the mechanisms underlying memory. Previous reports have shown that ECM molecules activated signal transduction pathways through diverse cell surface receptors. ${ }^{1}$ For example, integrins, the primary laminin receptors, are expressed in the adult hippocampus $^{30}$ and are involved in the stabilization of LTP. ${ }^{31}$ In this paper, we have shown that Timp-3 KO mice showed increased MMP activity in the hippocampus and impaired cognitive function compared with WT mice. Similarly, Timp-1deficient mice also showed learning and memory disturbances. $^{23,24}$ This result could also be explained by the fact that Timp-1 is expressed in the hippocampus, and synaptic plasticity is influenced by an increase in MMP activity. Timp2 is also suggested to be involved in synaptic plasticity underlying learning and memory. ${ }^{25}$
We conducted behavioral tests and brain tissue analyses in Timp-3 KO mice to clarify the involvement of Timp-3 in cognitive function. Three behavioral tests were used to evaluate memory function, and an open-field test was used to evaluate habituation. We detected a decline in memory function in $\mathrm{KO}$ mice in the water maze test with the invisible platform, but not in passive or active avoidance tests.

Immunostaining showed the presence of Timp-3 in the hippocampus, which is considered to be the main brain region involved in memory. Moreover, in situ zymography showed that the hippocampi of Timp-3 KO mice had more gelatinolytic activity than did WT mice, indicating that MMP activity was deregulated in Timp-3 KO mice. Whether hippocampal deregulation of enzymatic activity in Timp-3 KO mice is directly or indirectly involved in delayed acquisition of memory in the water maze test remains to be elucidated. Moreover, it is not known whether other regions of the brain contribute to the abnormality observed in Timp-3 KO mice. Consistent with a previous report, Timp-3 expression was also detected in the choroid plexus. ${ }^{32}$

During central nervous system development, Timp-3 is expressed in the embryonic ventricular zone and postnatal subventricular zone, where neurogenesis occurs. ${ }^{33}$ In addition, Timp-3 is expressed in the rostral migratory stream; a sub-population of cells in the subventricular zone migrates along the rostral migratory stream to the olfactory bulb, where cells differentiate into neurons. It is possible that lack of Timp-3 expression during brain development might have long-term effects on cognition, given that impaired cognitive function was observed in adult Timp-3 KO mice in this study.

In the water maze test using a visible platform, Timp-3 KO mice were able to use visual cues to reach the platform. However, in humans mutations in the Timp-3 gene result in SFD, a disease characterized by the loss of central vision during the fourth or fifth decade of life. A possible explanation for the discrepancy is that the Timp-3 KO mice we used were relatively young ( 3 months old). Alternatively, the difference in the nature of genetic alterations in SFD patients versus Timp-3 $\mathrm{KO}$ mice is another possibility. Most mutations observed in the Timp-3 gene in SFD patients involve either the introduction of a new cysteine residue in the C-terminal domain or the presence of an odd number of cysteine residues because of the introduction of a stop codon. ${ }^{34}$ Some of these SFD mutations may result in production of higher molecular-weight protein complexes, possibly dimers. ${ }^{35}$ Dimerized Timp-3 protein has an active role in the SFD disease process by accumulating in the eyes. ${ }^{35}$ In a study of eye tissues obtained from SFD patients, the thickened Bruch's membrane was strongly Timp-3 positive, except for sites where the retinal pigment epithelial cells, which normally produce Timp-3, had degenerated. ${ }^{36}$ In contrast, Timp-3 KO mice do not express Timp-3 transcripts, do not synthesize Timp-3 protein, and do not accumulate Timp-3 protein in the eye tissue. On the basis of these observations, 
we do not find it too surprising that Timp-3 KO mice were able to recognize visual cues.

The results of the open-field test indicated that habituation was functional in WT mice, but seemed to be lacking in $\mathrm{KO}$ mice. This may be because $\mathrm{KO}$ mice were unable to remember the environment over time, and thus could not habituate during the test period. It is also possible that the KO mice had a neurological disorder that will impair both memory and habituation.

Timp-3 has been shown to have various functions in previous reports that described other phenotypes of Timp-3 KO mice. For example, at the age of 21 months, Timp-3 KO mice exhibited left ventricular enlargement similar to that observed in dilated cardiomyopathy, cardiac muscle cell hypertrophy, and contractile dysfunction. ${ }^{37}$ Others reported that Timp-3 KO mice exhibited a spontaneous air space enlargement in the lung, and enhanced collagen degradation in the peribronchiolar space. ${ }^{38}$ In addition, Timp-3 was reportedly involved in apoptosis, cell proliferation, inhibition of cell proliferation, and angiogenesis. However, this study showed for the first time that Timp-3 deficiency impaired cognitive dysfunction, potentially through the deregulation of ECM homeostasis within the brain.

\section{ACKNOWLEDGEMENTS}

We thank Ms Taeko Kaimoto for excellent secretarial and technical assistance. This study was supported by a grant in aid for Scientific Research from the Ministry of Education, Science, Sports, and Culture, Japan, and by a grant from the Japan Foundation for Applied Enzymology.

\section{DISCLOSURE/CONFLICT OF INTEREST}

The authors declare no conflict of interest.

1. Dityatev A, Schachner M. Extracellular matrix molecules and synaptic plasticity. Nat Rev Neurosci 2003;4:456-468.

2. Dityatev A, Schachner M. The extracellular matrix and synapses. Cell Tissue Res 2006;326:647-654

3. Wright JW, Harding JW. The brain angiotensin system and extracellular matrix molecules in neural plasticity, learning, and memory. Prog Neurobiol 2004;72:263-293.

4. Nagase H, Woessner Jr JF. Matrix metalloproteinases. J Biol Chem 1999;274:21491-21494.

5. Milward EA, Fitzsimmons C, Szklarczyk A, et al. The matrix metalloproteinases and CNS plasticity: an overview. J Neuroimmunol 2007;187:9-19.

6. Firestein GS. Evolving concepts of rheumatoid arthritis. Nature 2003;423:356-361.

7. Tsunemi $K$, Takai S, Nishimoto $M$, et al. Possible roles of angiotensin IIforming enzymes, angiotensin converting enzyme and chymase-like enzyme, in the human aneurysmal aorta. Hypertens Res 2002;25: 817-822.

8. Iwashima $\mathrm{Y}$, Horio $\mathrm{T}$, Kuroda $\mathrm{S}$, et al. Influence of plasma aldosterone on left ventricular geometry and diastolic function in treated essential hypertension. Hypertens Res 2002;25:49-56.

9. Higuchi $M$, Yasuda $O$, Kawamoto $H$, et al. Tissue inhibitor of metalloproteinase-3 deficiency inhibits blood pressure elevation and myocardial microvascular remodeling induced by chronic administration of Nomega-nitro-L-arginine methyl ester in mice. Hypertens Res 2007;30:563-571.

10. Brew K, Dinakarpandian D, Nagase $H$. Tissue inhibitors of metalloproteinases: evolution, structure and function. Biochim Biophys Acta 2000;1477:267-283.
11. Leco KJ, Khokha R, Pavloff $N$, et al. Tissue inhibitor of metalloproteinases-3 (TIMP-3) is an extracellular matrix-associated protein with a distinctive pattern of expression in mouse cells and tissues. J Biol Chem 1994;269:9352-9360.

12. Fedak PW, Verma $\mathrm{S}$, Weisel RD, et al. Cardiac remodeling and failure: from molecules to man (Part II). Cardiovasc Pathol 2005;14:49-60.

13. Lambert E, Dasse $E$, Haye $B$, et al. TIMPs as multifacial proteins. Crit Rev Oncol Hematol 2004;49:187-198.

14. Nagase $H$, Visse R, Murphy G. Structure and function of matrix metalloproteinases and TIMPs. Cardiovasc Res 2006;69:562-573.

15. Dzwonek J, Rylski M, Kaczmarek L. Matrix metalloproteinases and their endogenous inhibitors in neuronal physiology of the adult brain. FEBS Lett 2004;567:129-135.

16. Agrawal SM, Lau L, Yong VW. MMPs in the central nervous system: where the good guys go bad. Semin Cell Dev Biol 2008; 19:42-51.

17. Yong VW, Power C, Forsyth P, et al. Metalloproteinases in biology and pathology of the nervous system. Nat Rev Neurosci 2001;2: 502-511.

18. Yong VW, Krekoski CA, Forsyth PA, et al. Matrix metalloproteinases and diseases of the CNS. Trends Neurosci 1998;21:75-80.

19. Park JB, Kwak HJ, Lee SH. Role of hyaluronan in glioma invasion. Cell Adh Migr 2008;2:202-207.

20. Ulrich R, Baumgartner W, Gerhauser I, et al. MMP-12, MMP-3, and TIMP-1 are markedly upregulated in chronic demyelinating theiler murine encephalomyelitis. J Neuropathol Exp Neurol 2006;65: 783-793.

21. Yong VW. Metalloproteinases: mediators of pathology and regeneration in the CNS. Nat Rev Neurosci 2005;6:931-944.

22. Ethell IM, Ethell DW. Matrix metalloproteinases in brain development and remodeling: synaptic functions and targets. J Neurosci Res 2007;85:2813-2823.

23. Jourquin J, Tremblay E, Bernard A, et al. Tissue inhibitor of metalloproteinases-1 (TIMP-1) modulates neuronal death, axonal plasticity, and learning and memory. Eur J Neurosci 2005;22: 2569-2578.

24. Chaillan FA, Rivera $S$, Marchetti $E$, et al. Involvement of tissue inhibition of metalloproteinases- 1 in learning and memory in mice. Behav Brain Res 2006;173:191-198.

25. Jaworski DM, Boone J, Caterina J, et al. Prepulse inhibition and fearpotentiated startle are altered in tissue inhibitor of metalloproteinase2 (TIMP-2) knockout mice. Brain Res 2005;1051:81-89.

26. Kawamoto $H$, Yasuda O, Suzuki T, et al. Tissue inhibitor of metalloproteinase-3 plays important roles in the kidney following unilateral ureteral obstruction. Hypertens Res 2006;29:285-294.

27. Amantea D, Corasaniti MT, Mercuri NB, et al. Brain regional and cellular localization of gelatinase activity in rat that have undergone transient middle cerebral artery occlusion. Neuroscience 2008; 152:8-17.

28. Mook OR, Van Overbeek C, Ackema EG, et al. In situ localization of gelatinolytic activity in the extracellular matrix of metastases of colon cancer in rat liver using quenched fluorogenic DQ-gelatin. J Histochem Cytochem 2003;51:821-829.

29. Upchurch $M$, Wehner JM. Differences between inbred strains of mice in Morris water maze performance. Behav Genet 1988;18: $55-68$.

30. Schuster $T$, Krug M, Stalder $M$, et al. Immunoelectron microscopic localization of the neural recognition molecules L1, NCAM, and its isoform NCAM180, the NCAM-associated polysialic acid, beta1 integrin and the extracellular matrix molecule tenascin- $R$ in synapses of the adult rat hippocampus. J Neurobiol 2001;49:142-158.

31. Staubli U, Chun D, Lynch G. Time-dependent reversal of long-term potentiation by an integrin antagonist. J Neurosci 1998;18: 3460-3469.

32. Pagenstecher $\mathrm{A}$, Stalder $\mathrm{AK}$, Kincaid $\mathrm{CL}$, et al. Differential expression of matrix metalloproteinase and tissue inhibitor of matrix metalloproteinase genes in the mouse central nervous system in normal and inflammatory states. Am J Pathol 1998;152:729-741.

33. Jaworski DM, Fager N. Regulation of tissue inhibitor of metalloproteinase-3 (Timp-3) mRNA expression during rat CNS development. J Neurosci Res 2000;61:396-408.

34. Li Z, Clarke MP, Barker MD, et al. TIMP3 mutation in Sorsby's fundus dystrophy: molecular insights. Expert Rev Mol Med 2005;7:1-15. 
35. Langton KP, McKie N, Curtis A, et al. A novel tissue inhibitor of metalloproteinases-3 mutation reveals a common molecular phenotype in Sorsby's fundus dystrophy. J Biol Chem 2000;275:27027-27031.

36. Fariss RN, Apte SS, Luthert PJ, et al. Accumulation of tissue inhibitor of metalloproteinases-3 in human eyes with Sorsby's fundus dystrophy or retinitis pigmentosa. Br J Ophthalmol 1998;82:1329-1334.
37. Fedak PW, Smookler DS, Kassiri Z, et al. TIMP-3 deficiency leads to dilated cardiomyopathy. Circulation 2004;110:2401-2409.

38. Leco KJ, Waterhouse $\mathrm{P}$, Sanchez $\mathrm{OH}$, et al. Spontaneous air space enlargement in the lungs of mice lacking tissue inhibitor of metalloproteinases-3 (TIMP-3). J Clin Invest 2001;108: 817-829. 